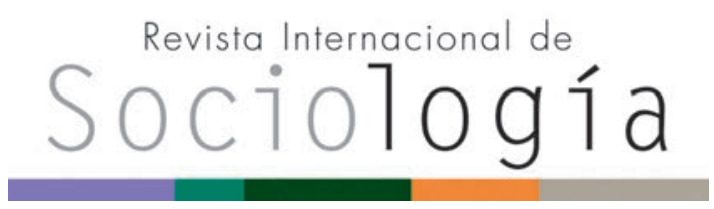

Revista Internacional de Sociología RIS vol. 75 (2), e059, abril-junio, 2017, ISSN-L:0034-9712 doi: http://dx.doi.org/10.3989/ris.2017.75.2.15.63

\section{DEL TRANSEXUALISMO A LA DISFORIA DE GÉNERO EN EL DSM. Cambios terminológicos, misma esencia patologizante}

\author{
JoRDI MAS GRAU \\ Universidad de Barcelona \\ jordimasgrau@gmail.com \\ ORCID iD: http://orcid.org/0000-0002-3414-9335
}

Cómo citar este artículo / Citation: Mas Grau, J. 2017. "Del transexualismo a la disforia de género en el DSM. Cambios terminológicos, misma esencia patologizante". Revista Internacional de Sociología 75 (2): e059. doi: http:// dx.doi.org/10.3989/ris.2017.75.2.15.63

\section{FROM TRANSSEXUALISM TO GENDER DYSPHORIA IN THE DSM. Terminological changes, same pathologising essence}

Copyright: (C) 2017 CSIC. Este es un artículo de acceso abierto distribuido bajo los términos de la licencia Creative Commons Attribution (CC BY) España 3.0.

Recibido: 15/04/2015. Aceptado: 13/05/2016. Publicado online: 24/05/2017

\begin{abstract}
This paper analyses how transsexuality has been con-ceptualized in the Diagnostic and Statistical Manual of Mental Disorders (DSM). We will see how the successive changes in the denomination and diagnostic criteria are largely due to the pressure that the editors of the manual have received by scientists, academics, political organ-isations and organisations for the transgender rights. As a result of these tensions the transsexuality has been reconceptualised in several occasions, although the con-notations inherent to the diagnostic category which treat it as a disease have endured. Likewise, we will address the current debate on pathologisation of transsexual-ity which is dominated by two opposed discourses: one which justifies the inclusion of transsexuality in the DSM because it considers that the diagnostic guarantees the access to hormonal and surgical therapy, and the other which criticises its inclusion because it considers that it contributes to the stigmatisation of the transgender peo-ple. The paper concludes that the trans-specific health resources should be regarded as a fundamental right which cannot be subject to clinical requirements.
\end{abstract}

\section{KEYWORDS}

Health; Psychiatry; Rights; Transgender;

Transsexuality. Derechos; Psiquiatría; Salud; Transexualidad;
Transgénero. 


\section{INTRODUCCIÓN}

La transexualidad entra en el Manual diagnóstico y estadístico de los trastornos mentales (DSM en sus siglas en inglés) de la Asociación Psiquiátrica Norteamericana (APA) en la tercera edición de 1980, debido en buena medida a la presión ejercida por la hoy denominada The World Professional Association for Transgender Health (WPATH). Esta asociación, con sede en Estados Unidos, fue creada en 1979 por un grupo de profesionales que trabajaban con personas transexuales. Con la inclusión de la transexualidad en el DSM, la WPATH quería abrir nuevas posibilidades legales y sociales para estas personas en Estados Unidos (Matte, Devor y Vladicka 2009). La tipificación de la transexualidad como un trastorno mental ha servido de fundamento para que el coste de la terapia de modificación corporal (basada en el tratamiento hormonal y las cirugías de reasignación sexual) sea asumido total o parcialmente por las aseguradoras privadas o por algunos sistemas sanitarios públicos (entre los que se encuentran los de algunas comunidades autónomas españolas ${ }^{1}$ ).

Con todo, desde el principio surgen voces que critican la presencia de la transexualidad en la considerada como "la Biblia de los profesionales de salud mental" (Earle 2014: 179). Asociaciones de personas "trans"2, defensores de los derechos civiles y profesionales y académicos de diversa índole sostienen que un diagnóstico psiquiátrico proporciona el terreno fecundo para la discriminación y el estigma social (Langer y Martin 2004; Useche 2005; Winters 2008; Lev 2013). Argumentan, asimismo, que el DSM opera como una herramienta de control social y legitimización del sistema de sexo/género, pues impide que se politice la insatisfacción de género al presentarla como una anormalidad patológica que tan solo concierne a la persona afectada y a los profesionales encargados de tratarla (Butler 2006; Nieto 2008, 2011). Por todo ello, a principios del siglo XXI se intensifica la presión internacional para que la transexualidad sea retirada del DSM, así como del capítulo "Trastornos mentales y del comportamiento" de la Clasificación Internacional de las Enfermedades (CIE) que elabora la OMS. Este movimiento recuerda inevitablemente al que logró, en 1973, la desclasificación de la homosexualidad del DSM.

En el presente artículo analizaremos el modo en que se ha conceptuado la transexualidad en el DSM. Prestaremos una atención especial a la "disforia de género", denominación que se da al fenómeno en la quinta y última edición del manual, aparecida en 2013. Mostraremos que los sucesivos cambios de denominación y de criterios diagnósticos que ha experimentado la transexualidad en el DSM reflejan las tensiones constantes entre, por un lado, los redactores del manual, y por el otro, académicos, organismos políticos y organizaciones "trans". Seguidamente, nos introduciremos en el debate habido entre los que defienden la inclusión de la transexualidad en las nosologías de trastornos mentales como una forma de garantizar la cobertura del tratamiento de modificación corporal, y aquellos que persiguen la desclasificación y buscan alternativas para que ello no afecte a los derechos de las personas "trans". En este sentido, presentaremos las principales demandas de la red internacional Stop Trans Pathologization, entre las que destaca la petición a la OMS para que en la próxima edición de la CIE se reconozca la atención sanitaria a este colectivo como un proceso de salud no basado en la enfermedad (de modo similar a lo que sucede con el embarazo).

\section{Consideraciones metodológicas Y EPISTEMOLÓGICAS}

Cuando abordemos el debate en torno a la patologización de la transexualidad, presentaremos algunos testimonios relevantes de personas "trans" y de profesionales médicos que trabajan con esta población, los cuales han sido recogidos durante una investigación que tenía por objeto analizar los discursos, representaciones y prácticas generados socialmente a lo largo del proceso de (re)construcción identitaria y corporal de las personas "trans" residentes en Cataluña. Uno de los principales objetivos de esta investigación, realizada entre 2009 y 2014, consistía en estudiar el proceso diagnóstico y terapéutico llevado a cabo en la Unidad de Trastornos de la Identidad de Género (UTIG) del Hospital Clínico de Barcelona $^{3}$. La principal técnica empleada fueron las entrevistas en profundidad semi-dirigidas a personas "trans" (en total, 13 entrevistas a hombres "trans" y 41 a mujeres "trans") y a profesionales sanitarios que trabajan con esta población (10 entrevistados, 7 de los cuales trabajan o habían trabajado en la UTIG).

Con las entrevistas a las personas "trans" se intentó que la muestra reflejara en la medida de lo posible tanto las múltiples y variadas formas de construir cuerpos y subjetividades, como las distintas opiniones existentes -a veces enfrentadas- sobre cuestiones tales como la necesidad de la diagnosis psiquiátrica (como requisito para acceder al tratamiento financiado por la sanidad pública catalana) y el cambio de sexo en los documentos y registros oficiales ${ }^{4}$. Como uno de los principales objetivos consistía en analizar la UTIG de Barcelona, se tenía especial interés en entrevistar a personas que eran usuarias de la Unidad. Para ello, fueron utilizadas dos estrategias. Primeramente, se pidió a la informante principal que trabajaba en la UTIG (una profesional de la medicina) que aprovechara las visitas médicas que realizaba para captar participantes. En segundo lugar, y con el fin de contactar directamente y sin intermediarios con estas personas, se acudió cuatro veces a las terapias de grupo que organizaba la UTIG mensualmente ${ }^{5}$. Además de las personas usuarias de la UTIG, se contactó con algunas de las principales organizaciones trans catalanas 
(Col-lectiu de Transsexuals de Catalunya, Associació de Transsexuals de Catalunya, Xarxa d'Acció TransIntersex y Cultura Trans) para entrevistar a personas provenientes del mundo asociativo.

Por otra parte, el análisis que se realiza del DSM (ediciones III, IV y V) se enmarca dentro de la corriente que estudia la capacidad de los saberes y discursos hegemónicos para producir subjetividades y significados sexo-genéricos (Plummer 1995; Hacking 1999; Foucault 2003; Butler 2007). En este sentido, las categorías diagnósticas referentes a la transexualidad no serán concebidas como entidades que reflejan fielmente un orden natural y objetivo, sino como tecnologías discursivas, es decir, como dispositivos de poder social y político que contribuyen decisivamente a la configuración de identidades (MartínezGuzmán e Î́niguez 2010). Efectuaremos, por tanto, un "análisis crítico del discurso", esto es, una forma de análisis que centra su atención en la forma en que el abuso del poder, el dominio y la desigualdad son reproducidos, y a veces combatidos, por los textos y el habla (Van Dijk 1999). En fin, nos alejaremos de una visión representacional del lenguaje para entenderlo como una práctica -sujeta a múltiples influjoscon capacidad para producir la realidad social (Austin 1982; Wittgenstein 1988; Rorty 1990). Partiendo del reconocimiento de la capacidad performativa del lenguaje, mostraremos que los discursos de la psiquiatría biomédica "no son meros reflejos de nuestra vida sexual, sino que desempeñan un papel activo en su construcción" (Plummer 1995: 12).

\section{Del transexualismo a la disforia de GÉNERO}

Como se ha comentado anteriormente, la transexualidad se incluye en el DSM en la tercera edición de 1980. Casi al mismo tiempo se excluye a la homosexualidad gracias, en gran medida, a la presión ejercida por los movimientos de gais y lesbianas, que llevaban tiempo reivindicando la despatologización del deseo homoerótico. Tanto el proceso de desclasificación de la homosexualidad, como los sucesivos cambios de denominación y de criterios diagnósticos que ha experimentado la transexualidad desde que fue incluida por vez primera en el DSM, constituyen una buena oportunidad para recuperar el concepto de "nominalismo dinámico" creado por Hacking (1999). Para este filósofo canadiense, la creación de una nueva categoría humana -como la homosexualidad o la transexualidad- tiene importantes efectos sobre las personas etiquetadas, puesto que cada categoría abre nuevas posibilidades de ser y de existir, configura un nuevo espacio para la autointeligibilidad. Sin embargo, las personas no aceptan de forma acrítica las nuevas categorías, ya que pueden resignificarlas o rechazarlas. Se produce así una constante interacción -aunque, a menudo, asimétrica- entre las personas y las formas en que son categorizadas.
$Y$ esto es justamente lo que sucede con el DSM y las personas tipificadas como "transexuales" u "homosexuales". La APA desarrolla categorías que influyen en las personas diagnosticadas, pues la fuerza y legitimidad que rodean al manual (y, por extensión, a la psiquiatría norteamericana y a la biomedicina en general) facilita el que las personas interioricen que su condición es anormal, patológica. Un ejemplo de ello lo encontramos en las palabras de Aurora ${ }^{6}$, una de las mujeres trans entrevistadas que era usuaria de la UTIG: "Igual que nacen personas ciegas o con síndrome de Down, nacen personas transexuales". Con todo, existen personas "trans" que rechazan la visión patologizante que se tiene de ellas. Estas personas han creado incluso un término autorreferencial, "transgénero", con el objetivo de alejarse de las categorías clínicas (transexual y travesti) con que estaban siendo diagnosticadas. Bajo el paraguas del vocablo "transgénero" encontramos a una multitud identitaria que expresa un género distinto al de asignación. Entre esta multitud hay personas que no quieren ajustarse a la lógica de género dicotómica y reniegan del protocolo asistencial estandarizado, basado en la terapia hormonal y las cirugías de reasignación sexual ${ }^{7}$.

Sin embargo, algunos estudios (Mas Grau 2015; Missé y Coll-Planas 2015) han mostrado que no solo el discurso biomédico produce una normatividad mediante la creación de categorías psiquiátricas, puesto que los discursos y categorizaciones del activismo crítico (como queer o transgénero) también pueden crear sus propias jerarquías en función del nivel de compromiso personal con el proyecto contestatario (estableciéndose diferencias entre auténticos transgresores y víctimas del sistema de género). Sea como fuere, los términos "travesti", "transgénero" y "transexual" han tenido usos y significados cambiantes en función del periodo histórico y el contexto sociocultural, tal y como revelan los trabajos realizados en España (Soley-Beltran y Coll-Planas 2011; Platero 2011; Soley-Beltran 2013) y en Estados Unidos (Valentine y Kulick 2001; Valentine 2007). El empleo y significado de estas categorías revela una compleja interacción entre instituciones políticas, organismos médicos y sanitarios, centros académicos y organizaciones trans, con la que se negocian constantemente significados sobre el género, la sexualidad y, por extensión, la realidad.

Pero lo que aquí nos interesa analizar es la reacción que ha tenido históricamente la APA ante las presiones para desclasificar la homosexualidad y, en especial, la transexualidad. Y es que este organismo siempre ha mostrado grandes reticencias a la hora de eliminar un diagnóstico de sus clasificaciones, por lo que antes tiende a realizar concienzudos esfuerzos de reconceptualización, lo que Nieto (2008) denomina "camouflage o travestissement semántico", con el objetivo de ofrecer una versión eufemística de la patología -a menudo, recurriendo a tecnicismossin modificar con ello su esencia patologizante. 
En el caso de la homosexualidad, en la séptima reimpresión del DSM-II (1974) los miembros de la APA deciden cambiar este término por "perturbación de la orientación sexual”. En el DSM-III (1980), sustituyen esta categoría por "homosexualidad egodistónica", un diagnóstico creado para referirse a aquellas personas que sufren a causa de su orientación homosexual. En la edición revisada del DSM-III (1987), se elimina este concepto por considerarse que dicho sufrimiento, de estar presente, es producto de la homofobia social y no de la condición homosexual per se (Braunstein 2005; Nieto 2011; Lev 2013). Pero la APA crea entonces, para el DSM-IV, los "trastornos sexuales no especificados", una categoría con la que puede ser diagnosticada toda aquella persona que siente una "sensación profunda de inadecuación con respecto a la actitud sexual u otros rasgos relacionados con los estándares autoimpuestos de masculinidad o feminidad" o un "malestar profundo y persistente en torno a la orientación sexual" (Asociación Psiquiátrica Norteamericana 2002: 247). Estos criterios diagnósticos nos sugieren que se ha creado una categoría paraguas con la que seguir "psiquiatrizando" a las expresiones sexo-genéricas no normativas y que no tienen un diagnóstico específico. No es difícil advertir que la "sensación de profunda inadecuación" o el "malestar profundo y persistente" se deben dar muy especialmente en aquellas personas que experimentan las sanciones sociales por tener una orientación sexual o desarrollar unos roles de género que no se ajustan a la lógica heteronormativa y androcéntrica.

La evolución de la transexualidad en el DSM presenta grandes paralelismos con el recorrido que ha tenido la homosexualidad. En el DSM-III, el fenómeno fue denominado "transexualismo". Los criterios diagnósticos de este nuevo trastorno reflejaban la influencia de los primeros teóricos, como Benjamin (1966) o Stoller (1968; 1975). Aparte de haber alcanzado la pubertad (a los niños se les diagnosticaba el "trastorno de la identidad sexual en la infancia"), eran necesarios dos requisitos más para confirmar el diagnóstico: un malestar persistente respecto al propio sexo anatómico y "una preocupación de por lo menos dos años de duración sobre cómo deshacerse de las características sexuales primarias y secundarias y de cómo adquirir las características sexuales del otro sexo" (APA 1989: 94). Con estos criterios se estaba validando el concepto de "transexual verdadero" (Benjamin 1966), cuyo principal rasgo definidor era la firme voluntad del sujeto de someterse a la cirugía genital. A aquellas personas que no cumplían con este requisito de autenticidad, se les reservaba otro diagnóstico: el "trastorno de la identidad sexual en la adolescencia o en la vida adulta". Como recuerda Mejía (2006), en aquella época las personas que no querían operarse eran vistas como pseudotransexuales.
Con la publicación del DSM-III surgen las primeras personas que muestran su incomodidad porque se está patologizando explícitamente su condición. Es por ello que la APA decide cambiar de denominación en la siguiente edición del manual dando un giro conceptual que recuerda inevitablemente al que experimentara la homosexualidad en 1974. Ya no se nombra a la homosexualidad ni a la transexualidad, pero se patologiza el deseo homoerótico y la identificación de género cruzada, con el empleo de términos que denotan enfermedad: "perturbación de la orientación sexual" y "trastorno de la identidad de género" (en adelante TIG). En opinión de Nieto (2008), los redactores del DSM podrían haber utilizado otras palabras que resaltaran la agencia del sujeto, como "disconformidad" o "rechazo" (de género), pero se decantan por "trastorno" al ser un término "enfermizante" que legitima la intervención psiquiátrica.

En el DSM-IV, el TIG está incluido en el apartado de los "trastornos sexuales y de la identidad sexual", que se dividen en cuatro tipos: las "disfunciones sexuales" ( $p$. ej. la eyaculación precoz o el deseo sexual hipoactivo), las "parafilias" (p. ej. el fetichismo o el exhibicionismo), los "trastornos de la identidad de género" (donde se incluye al TIG) y el "trastorno sexual no especificado" (esa categoría paraguas de la que hablábamos anteriormente). Para poder diagnosticar el TIG, se requiere el cumplimiento de los siguientes criterios: "A. Identificación acusada y persistente con el otro sexo"; "B. Malestar persistente con el propio sexo o sentimiento de inadecuación con su rol"; "C. La alteración no coexiste con una enfermedad intersexual"; "D. La alteración provoca malestar clínicamente significativo o deterioro social, laboral o de otras áreas importantes de la actividad del individuo" (Asociación Psiquiátrica Norteamericana 2002: 245-246). Una de las principales novedades respecto a la edición anterior es la eliminación del trastorno específico para aquellas personas que no muestran un deseo persistente de modificar sus caracteres sexuales. Aun así, los criterios establecidos para el TIG siguen asociando estrechamente la transexualidad con el malestar corporal y el deseo de pasar por el quirófano:

\footnotetext{
"En los adolescentes y en los adultos la alteración se manifiesta por síntomas como preocupación por eliminar las características sexuales primarias y secundarias (p. ej., pedir tratamiento hormonal, quirúrgico $\mathrm{u}$ otros procedimientos para modificar físicamente los rasgos sexuales y de esta manera parecerse al otro sexo) o creer que se ha nacido con el sexo equivocado" (ibídem: 246).
}

Si nos fijamos en los criterios diagnósticos A y B, estos tan solo adquieren sentido si presuponemos que únicamente es normal la total correspondencia entre el sexo biológico y la identidad de género (Useche 2005). De este modo, cualquier persona que rechaza el género que se le asigna al nacer y 
desea adoptar una apariencia socialmente vinculada con el otro género, es susceptible de padecer un trastorno. Esto supone un claro reforzamiento de uno de los postulados centrales de nuestro sistema de sexo/género, según el cual ha de existir una estrecha correlación entre los caracteres sexuales y la identificación de género de la persona (Mas Grau 2013). La APA afirma que se limita a ofrecer "la mejor descripción que existe sobre cómo se presentan los trastornos mentales y cómo pueden los clínicos reconocerlos" (Asociación Psiquiátrica Norteamericana 2014: XLV). El enfoque eminentemente descriptivo del DSM parece obedecer a una voluntad científica desapasionada, neutral, alejada de prejuicios. No obstante, esta "asepsia" epistemológica no parece tal cuando observamos los criterios diagnósticos del TIG, en los que lo normativo también tiene cabida. El sistema clasificatorio del DSM se basa tanto en datos científicos como en valores sociales, ya que la concepción de normalidad que se sostiene en este manual se basa en buena medida en nociones de conformidad social (Klerman 1987; Laungani 2002; Martínez y Montenegro 2010).

Además, sostener que estas personas se identifican "con el otro sexo" supone una simplificación de una realidad que es plural y diversa. Esta lógica dicotómica ignora que existen personas cuyos cuerpos y subjetividades no se ajustan al binomio hombre/ mujer y que cuestionan las categorías identitarias que conforman nuestro universo sexo-genérico. En este sentido, las palabras de Luis (activista) reflejan este posicionamiento crítico: "No se valora el tránsito. ¿Por qué se ignora a la gente que transita, es decir, a aquellos que no se sienten ni hombre ni mujer? No hay que construir constantemente hombres y mujeres transexuales".

Cuando se acaba de publicar la edición revisada del DSM-IV, en el año 2002, ya son varios los ámbitos desde los que se pone en duda la inclusión de la transexualidad en las nosologías de los trastornos mentales (Nieto y De la Calle 2015). Estas voces críticas provienen incluso del mismo grupo de trabajo encargado de la revisión de los "trastornos de la identidad de género" para el DSM-5, como CohenKettenis y Pfäfflin (2010), quienes cuestionan que la divergencia entre el género asignado y la identidad de género sea, por sí misma, un trastorno mental. A ello debemos añadirle que, a finales de los años 2000 , autoridades políticas y organismos internacionales empiezan a posicionarse a favor de la despatologización. Es el caso del Comisario Europeo de Derechos Humanos, Thomas Hammarberg, quien en un informe de 2009 solicita la desclasificación de la transexualidad al considerar que la atención sanitaria puede realizarse sin efectuar antes un diagnóstico de trastorno mental. Por su parte, en una resolución de septiembre de 2011, el Parlamento europeo exige la "desiquiatrización de la vivencia transexual y trans- género" (punto 13) y pide a la OMS que, en la undécima versión de la CIE (prevista para 2018), suprima "los trastornos de identidad de género de la lista de trastornos mentales y del comportamiento, y que garantice una reclasificación de dichos trastornos como trastornos no patológicos" (punto 16). En fin, en junio de 2012 el pleno del Parlament de Catalunya incluye en una declaración institucional la necesidad de perseverar en el proceso de revisión de la próxima edición de la CIE para que se excluya al transexualismo del catálogo de enfermedades mentales.

Ante esta coyuntura, la APA tenía que reaccionar de algún modo. La voluntad de mostrarse sensibles ante los comentarios y críticas recibidos llevó a la APA a reconceptualizar de nuevo la categoría diagnóstica ${ }^{8}$. En un primer borrador, publicado en $2010^{9}$, cambiaron el "trastorno de la identidad de género" por "incongruencia de género" (Gender Incongruence), afirmando que comprendían las objeciones de las asociaciones en torno al uso de la palabra "trastorno" como elemento estigmatizante. La APA sostenía que la nueva categoría reflejaba mejor la "esencia del problema" porque permitía centrar la atención en "la incongruencia existente entre la identidad que uno experimenta o expresa y el género asignado".

A pesar de este cambio, las críticas no remitieron puesto que el uso del término "incongruencia" conlleva también una fuerte carga estigmatizante. Baste recordar que la RAE define esta palabra, en su segunda acepción, como "un dicho o hecho faltos de sentido o de lógica”. Asimismo, la APA se había planteado situar esta categoría diagnóstica en un apartado especial del manual, al ser "un tipo inusual de trastorno mental que es tratado con hormonas y cirugías de reasignación sexual". Aunque al final optaron por no destacar su excepcionalidad por la misma razón con la que justifican su no desclasificación: si bien reconocen que el diagnóstico "puede tener un efecto estigmatizante", destacan que, al mismo tiempo, "facilita la asistencia clínica y la cobertura del seguro médico" (Asociación Psiquiátrica Norteamericana 2013a). Así pues, la transexualidad sigue en el DSM para no poner en peligro el acceso al tratamiento de estas personas.

Pero en la versión definitiva del DSM-5 han realizado otro cambio terminológico y se han decantado por "disforia de género", argumentando que "incongruencia de género" es una categoría "que podría aplicarse erróneamente a personas con conductas de género atípicas pero que, en cambio, no tienen ningún problema de identidad de género". La APA se inclina finalmente por esta denominación por tener "una larga historia en la sexología clínica y resultar familiar a clínicos y especialistas en el tema". El concepto "disforia de género" fue acuñado por el médico inglés Norman Fisk (1974) para referirse no solo a la transexualidad sino también a otros trastornos relacionados con la identidad de género. Con el tér- 
mino "disforia", Fisk pretendía destacar el malestar personal resultante del conflicto entre la identidad de género y el sexo biológico, malestar que adquiriría su grado máximo en el caso de la transexualidad.

Con el cambio de denominación, la esencia del diagnóstico ya no es la identificación de género cruzada (la APA admite que la no conformidad de género no es per se un trastorno mental), sino "el malestar que puede acompañar a la incongruencia entre el género experimentado o expresado y el género que se asigna" (Asociación Psiquiátrica Norteamericana 2014: 451). No obstante, convertir el malestar (o su versión técnica "disforia") en sinécdoque de la categoría diagnóstica supone otra forma más de homogeneizar la pluralidad que caracteriza al mundo "trans". Existen personas que no sienten angustia alguna por su condición. Y si en realidad experimentan algún malestar, éste es generado por una sociedad tránsfoba que las estigmatiza. Además, el empleo de esta categoría conlleva ampliar el alcance de los sujetos diagnosticables, pues no pocos gais y lesbianas sienten malestar -o rechazo- hacia un género asignado en base a los principios de la heteronormatividad. Y si concebimos el género como un ideal normativo de difícil personificación, podemos sostener que el concepto de disforia "es tan amplio que, posiblemente, todas las personas la experimentamos de forma más o menos leve" (King 1993: 64).

\section{LA DISFORIA DE GÉNERO}

Los cambios efectuados en esta última edición no se limitan al nombre del diagnóstico, sino que también afectan a la ubicación del trastorno dentro del manual, a los criterios diagnósticos y a los especificadores. En cuanto a la ubicación, es de destacar que la "disforia de género" forma una nueva clase diagnóstica dentro del DSM-5, por lo que ha sido separada de las "disfunciones sexuales" y las "parafilias". Esta reclasificación puede ser entendida como otro intento más de la APA para lograr una categoría de apariencia menos estigmatizante, pues ahora ya no está junto al "exhibicionismo" o la "pedofilia". En relación a los criterios diagnósticos, se ha decidido tratar separadamente la disforia infantil de la disforia durante la adolescencia y la adultez. Centrémonos en los criterios diagnósticos de esta última:

\footnotetext{
"A. Una marcada incongruencia entre el sexo que uno siente o expresa y el que se le asigna, de una duración mínima de seis meses, manifestada por un mínimo de dos de las características siguientes:
}

1. Una marcada incongruencia entre el sexo que uno siente o expresa y sus caracteres sexuales primarios o secundarios (o en los adolescentes jóvenes, los caracteres sexuales secundarios previstos).

2. Un fuerte deseo por desprenderse de los caracteres sexuales propios primarios o secun- darios, a causa de una marcada incongruencia con el sexo que se siente o expresa (o en los adolescentes jóvenes, un deseo de impedir el desarrollo de los caracteres sexuales secundarios previstos).

3. Un fuerte deseo por poseer los caracteres sexuales, tanto primarios como secundarios, correspondientes al sexo opuesto.

4. Un fuerte deseo de ser del otro sexo (o de un sexo alternativo distinto del que se le asigna).

5. Un fuerte deseo de ser tratado como del otro sexo (o de un sexo alternativo distinto del que se le asigna).

6. Una fuerte convicción de que uno tiene los sentimientos y reacciones típicos del otro sexo (o de un sexo alternativo distinto del que se le asigna).

B. El problema va asociado a un malestar clínicamente significativo o a un deterioro en lo social, laboral u otras áreas importantes del funcionamiento" (APA 2014: 452-453).

Antes de analizar estos criterios, hay que abordar la traducción al español que se ha efectuado de esta categoría, puesto que el uso que se hace del término "sexo" es, cuando menos, conflictivo. Una de las principales novedades que introduce la edición original en inglés del DSM-5 con respecto a su predecesora, es el empleo generalizado del término "género" (gender) cuando se habla del deseo o la experiencia del sujeto. El criterio A.4 de la edición original empieza del siguiente modo: " $A$ strong desire to be of the other gender [...]" (APA 2013b: 452). También podemos encontrar expresiones tales como: "experienced/expressed gender", "assigned gender" o "some alternative gender". Como podemos comprobar si leemos los criterios de la edición española (la empleada más arriba), los traductores han decidido sustituir "género" por "sexo". Sorprende que los traductores hayan eliminado por completo la palabra "género", ya que existe actualmente un amplio consenso, incluso en el mundo hispanohablante, sobre el uso de este término al referirse a la significación social y personal de la morfología corporal. Una hipótesis plausible que explicaría esta decisión sería que los traductores de la versión en español han decidido alinearse con aquellos que consideran que el grupo de trabajo encargado de los "trastornos de la identidad de género" para el DSM-5 ha incurrido en un exceso de sociologismo. Es el caso de Lawrence $(2010,2014)$, quien lamenta que en la nueva edición se haya privilegiado "el género socialmente construido antes que el esencialismo del sexo biológico" (Lawrence 2014: 1264). A modo de ejemplo, esta autora critica que se haya abandonado la definición clásica de la transexualidad (an incongruence between assigned sex and gender identity) por una versión (an incongruence between assigned gender and experienced/expressed gender) en la que el 
sexo -biológico- pierde su protagonismo en beneficio del género. Lawrence sospecha que este cambio no obedece a razones científicas sino políticas, esto es, a los esfuerzos de la APA por presentarse como un organismo "más progresista y abierto" ante las tesis de algunos científicos sociales y organizaciones.

Uno de los principales cambios respecto al DSMIV es la fusión de los anteriores criterios A ("identificación acusada y persistente con el otro sexo") y B ("malestar persistente con el propio sexo o sentimiento de inadecuación con su rol"), debido a que la APA ha considerado que su separación no estaba avalada por estudios empíricos. En cuanto al nuevo criterio $A$ (y el subcriterio A.1), salta a la vista el empleo del término "incongruencia" a pesar de haberlo excluido finalmente de la denominación del diagnóstico. De forma similar a lo que sucede con la palabra "trastorno", se puede dudar de la voluntad meramente descriptiva de la APA, pues ha vuelto a emplear un vocablo que connota anormalidad (ese "hecho falto de sentido o de lógica") en lugar de otros más neutros o empoderantes como, por ejemplo, "discrepancia".

Otra novedad interesante es el establecimiento de un periodo mínimo de seis meses para determinar la "marcada incongruencia". En uno de los borradores previos, los miembros del grupo de trabajo explican que la literatura científica no establece ningún umbral idóneo, pero que al final han decidido consensuar estos seis meses para poder realizar una "mínima distinción" entre la disforia transitoria y la persistente. A este respecto, todo parece indicar que la fijación de un periodo específico obedece al deseo de contrarrestar, mediante cuantificación, las fronteras difusas que rodean a la mayoría de trastornos mentales. En un artículo en el que participa el presidente del comité elaborador del DSM-5 (David J. Kupfer), se subraya la necesidad de establecer umbrales de normalidad/ patología del mismo modo que se establecen para la presión sanguínea o el colesterol, "ya que estos umbrales ayudan a los clínicos a detectar patologías con mayor exactitud y a determinar una intervención apropiada" (Kupfer, Kuhl y Regier 2013: 1691).

Por otra parte, hay que reconocer que la APA muestra en el DSM-5 una mayor receptividad ante los debates teórico-conceptuales impulsados por las ciencias sociales y el activismo de género. En la introducción al diagnóstico se habla por vez primera de "transgénero" y se recurre a una definición que goza de cierto consenso: el término "se refiere al amplio espectro de sujetos que de forma transitoria o permanente se identifica con un género diferente de su género natal" (APA 2014: 451). Asimismo, con los subcriterios A.4, A.5 y A.6 han abandonado el lenguaje binario para pasar a reconocer la existencia de géneros alternativos al binomio hombre/mujer. Otro aspecto a resaltar es que, al haber establecido el cumplimiento de un mínimo de dos subcriterios, ya no es necesario mostrar el deseo de operarse los genitales para obtener el diagnóstico. Con estas últimas modificaciones se han relajado las exigencias para aquellas personas que no se ajustan al ideal transexual. Ello constituye un hecho positivo si seguimos a la APA y entendemos el diagnóstico como el principal garante para el acceso a un tratamiento financiado por la sanidad pública o privada. Aunque también podemos preguntarnos si esta laxitud no conlleva un aumento del radio de acción patologizante sobre la diversidad "trans".

Si nos fijamos en el criterio $B$, se dice que el trastorno provoca no solo malestar, sino también "deterioro en lo social, laboral u otras áreas importantes del funcionamiento". En el borrador de 2010, los miembros del grupo de trabajo recomendaban que el diagnóstico se realizara sobre la base del criterio $A$, y que se evaluara el criterio $B$ de forma separada e independiente porque numerosos estudios demostraban que el malestar y el deterioro no eran rasgos inherentes a la transexualidad, sino más bien efectos de la transfobia. Indicaban además que este criterio estaba siendo evaluado por el órgano coordinador de todos los grupos de trabajo, puesto que aparece literalmente en varias de las categorías del DSM. Pero en la edición definitiva nada ha cambiado: este criterio tiene la misma fuerza que el A y nada evita suponer que es el trastorno en sí el que genera el deterioro social del individuo. Con la permanencia de este criterio se convierte una problemática de orden social, una situación de exclusión social, en un criterio diagnóstico individualizado. Hecho que impide realizar una lectura en clave social de la transexualidad para presentarla en cambio como un fenómeno que atañe únicamente a la estabilidad mental y social de la persona (Mas Grau 2013).

En lo referente a los subtipos y especificadores, se han eliminado los dos que constaban en el DSMIV: la edad del sujeto (ya que ahora se diagnostica separadamente a los niños de los adolescentes y adultos) y la orientación sexual. En su lugar, se establece la necesidad de especificar si el diagnóstico coexiste con un "trastorno del desarrollo sexual" y se añade el subtipo "postransición", esto es, determinar si la persona ya vive a tiempo completo en el género deseado y ha iniciado alguna terapia de modificación corporal. La explicación de la APA respecto a la inclusión de este último subtipo concuerda con la razón esgrimida para justificar la clasificación de la transexualidad: "asegurar el acceso al tratamiento a aquellas personas que continúan sometiéndose a la terapia hormonal, las cirugías o la psicoterapia" (APA 2013a). Por otro lado, la inclusión del primer subtipo resulta mucho más problemática. Hay que recordar que en el DSM-IV existía un criterio diagnóstico (el C) que estipulaba que el TIG solo podía diagnosticarse "si la alteración no coexiste con una enfermedad intersexual" (Asociación Psiquiátrica Norteamericana 2002: 246). Con el DSM-5, en cambio, las personas 
intersexuales pueden ser diagnosticadas con "disforia de género" si el género que sienten como propio no coincide con el género que el estamento médico les adjudicó en la infancia (y que a menudo fue consolidado con agresivas cirugías). Por lo que, aparte de estar fuertemente medicalizadas, estas personas ven ahora como el rechazo del género médicamente impuesto se incluye en un manual de trastornos mentales. Como denuncian desde la campaña Stop Trans Pathologization (2010): "Si a un infante intersexual se le asigna un género sin su consentimiento, cuando quiera ejercer su propia opción de asignación es inaceptable que ésta sea considerada como un trastorno psicológico".

La última novedad remarcable del capítulo sobre la "disforia de género" es la inclusión de un apartado sobre los "aspectos diagnósticos relacionados con la cultura"10. Lo primero que salta a la vista es el escaso peso que tiene este apartado: ocupa, literalmente, 4 líneas de las 9 páginas que conforman el capítulo. Una extensión que parece a todas luces suficiente si tenemos en cuenta que la mayoría de elementos socioculturales intervinientes en la configuración de la "disforia de género" han sido previamente transformados -o, más bien, "desculturizados"- en criterios diagnósticos. Esta no es característica exclusiva de esta categoría diagnóstica, puesto que a lo largo del DSM los trastornos mentales son aprehendidos como si fueran entidades orgánicas y tratados del mismo modo que las enfermedades físicas, mientras que los factores psicosociales y socioculturales son convertidos en criterios diagnósticos que parecen responder a un orden de realidad universal y reconocible (Martínez Hernáez 2000; Laungani 2002). Con el fin de analizar el contenido de los "aspectos culturales" vinculados a la disforia de género, merece la pena reproducir lo dicho en este apartado:

\begin{abstract}
"Se han descrito sujetos con disforia de género en muchos países y culturas. También se ha descrito un equivalente a la disforia de género en sujetos que viven en culturas con otras categorías sexuales institucionalizadas distintas del hombre y la mujer. No está claro si en estos sujetos se cumplirían los criterios diagnósticos de disforia de la disforia de género"

(Asociación Psiquiátrica Norteamericana 2014: 457).
\end{abstract}

Si nos fijamos en las tres frases del párrafo podemos constatar que su tono oscila entre la confianza y la cautela. A la habitual confianza en la aplicabilidad transcultural del diagnóstico (el fragmento "en muchos países y culturas" lo encontramos en otras categorías del manual) se le une la duda de aplicarlo a personas -que pertenecen a una otredad cultural- con expresiones de género alternativas. En este sentido, la segunda y la tercera frase son algo imprecisas. Han detectado algo equivalente a la disforia de género en sociedades con una estructura genérica no bipolar, pero no especifican en qué consiste esa equivalencia que no se atreven a diagnosticar. Sin embargo, podríamos suponer que aquello que no se nombra son figuras transgenéricas como el mahu polinesio o el hijra indio. De ser así, la aplicación de esta categoría diagnóstica es de lo más cuestionable porque estas figuras no tienen como principal elemento definidor el malestar -o disforia- con el que la psiquiatría caracteriza al transexual medicalizado. Aunque bien es cierto que, actualmente, muchos hijras y mahus sufren el deterioro de su posición social con la progresiva pérdida de su significación simbólica, causada, en gran medida, por la mundialización de los códigos sexogenéricos occidentales y del paradigma biomédico de la transexualidad (Nieto 2008, 2011). Respecto a la primera frase de la cita, Margaret Mead nos ofrece una explicación de lo más certera para entender que, efectivamente, el malestar (o, si queremos, la disconformidad o rechazo) de género se da "en muchos países y culturas". Aunque si para el DSM ese malestar es una cualidad inmanente de la persona, para la antropóloga norteamericana hemos de buscar las causas que lo generan en el universo social:

\begin{abstract}
"Cualquier sociedad que especializa sus tipos de personalidad según el sexo, que insiste en que cualquier rasgo -amor a los niños, interés en el arte, valor frente al peligro, locuacidad, falta de interés en las relaciones personales, pasividad en las relaciones sexuales; hay cientos de rasgos que han sido especializados así- está inalienablemente unido al sexo, prepara el camino que conduce a inadecuaciones del peor orden (Mead 2006: 271).
\end{abstract}

\section{DEBATES Y PROPUESTAS EN TORNO A LA DESPATOLOGIZACIÓN DE LA TRANSEXUALIDAD}

El debate sobre la presencia de la transexualidad en las nosologías de los trastornos mentales está dominado por dos discursos contrapuestos. Por un lado, parte del estamento médico defiende que el diagnóstico es la mejor garantía para asegurar el acceso a los recursos sanitarios de las personas "trans". Esta opinión no solo es sostenida por los redactores del DSM, sino también por la mayoría de profesionales médicos que trabajan en la UTIG estudiada:

\footnotetext{
"Yo siempre les digo a los transexuales que, al margen de las consideraciones filosóficas, peleen por que sigan estando allí (en los manuales). El hecho de que figuren en alguna clasificación diagnóstica es lo que burocráticamente permite a la sanidad pública hacerse cargo de la asistencia" (Profesional UTIG).
}

Pero entre la clase médica también existen profesionales (incluso dentro de la UTIG) que cuestionan la clasificación de la transexualidad en los manuales. Algunos de ellos entienden que el malestar -disforia- que actualmente define al fenómeno en el DSM es más el producto de la exclusión social que una propiedad intrínseca de la persona. Entre los que ponen en duda su presencia en las nosologías psiquiátricas, está muy presente el proceso de retirada del manual que siguió la homosexualidad: 
"Para poder entrar en las clasificaciones, las enfermedades han de crear malestar, eso es lo que está establecido. Pero pienso que un homosexual, al principio, tiene el malestar de aceptar esta situación porque en la sociedad está establecido el tema de la heterosexualidad. Hay muchas situaciones en la vida que crean malestar. La homosexualidad crea malestar pero se ha retirado de las clasificaciones. Creo que el malestar que crea la transexualidad es como cualquier otro: como tener un padre que realiza abusos sexuales. Es un malestar que puede tener cualquiera en diferentes circunstancias". (Psiquiatra que ha trabajado en una UTIG).

Entre las personas entrevistadas también existen opiniones encontradas. Algunas comparten el razonamiento de tipo estratégico sostenido por la clase médica para no eliminar la transexualidad de los manuales. Hay que destacar que la decisión de algunas comunidades autónomas españolas de financiar las cirugías de reasignación genital ha sido posible gracias a los que han defendido el carácter no electivo de este tipo de cirugías. $Y$ dicha defensa se ha fundamentado recurriendo a la presencia de la transexualidad en el DSM o la CIE y destacando el malestar sufrido por estas personas. Si además tenemos en cuenta que la sanidad pública española ha sufrido importantes recortes presupuestarios en los últimos años, y que desde algunos sectores conservadores se ha puesto la atención pública a la transexualidad como ejemplo de gasto superfluo a eliminar ${ }^{11}$, no es difícil entender que algunas personas se resistan a un cambio de paradigma: "Aunque no me guste que nos vean como enfermos mentales, creo que ahora no es el momento para tener estos debates. Hemos de tener mucho cuidado con lo que decimos porque están cerrando quirófanos por todas partes" (Jon, usuario de la UTIG).

Aun así, la mayoría de personas entrevistadas defienden la desclasificación. Estas personas opinan que nada justifica la patologización e insisten con vehemencia en que no padecen ningún trastorno mental: "He tenido una vida organizada, soy madura, me he pagado mis estudios y mi piso con mi trabajo. No soy una persona con problemas mentales, no estoy trastornada" (Andrea, usuaria de la UTIG). Uno de los argumentos más utilizados es que el diagnóstico consolida el estigma y el rechazo social: "El hecho de que esté dentro del ámbito de la psiquiatría socialmente queda como si fuéramos unos enfermos" (Dani, usuario de la UTIG). Desde este posicionamiento crítico, se recuerda que la disforia no caracteriza la vida de todas las personas y se sostiene que el principal problema sobre el que actuar no es la transexualidad en sí misma, sino la transfobia social: "Muchas vivimos nuestro cuerpo con total normalidad y disfrutamos de nuestros genitales como cualquier persona (...) Si alguien ha de ser visto como un enfermo o tiene que someterse a una prueba para saber si es normal, es la sociedad, no yo" (Regina, activista trans). Por todo ello, la enésima mutación terminológica realizada en el DSM no satisface a estas personas, para las que resulta innegociable la eliminación de toda categoría que sirva para patologizar su condición:

\begin{abstract}
"Este cambio de nombre es un engaño. Sirve para que los que escriben estos manuales se presenten como más progresistas, pero en realidad siguen viéndonos como a unos bichos raros y entonces la sociedad hace lo mismo. Si realmente creen que la transexualidad no es un trastorno, pues que la eliminen del manual" (Mónica, activista trans).
\end{abstract}

En el terreno del activismo, organizaciones de todo el mundo trabajan hoy en día para que la transexualidad sea eliminada de los manuales clasificatorios de los trastornos mentales y buscan alternativas para que la desclasificación no afecte a sus derechos sanitarios. Muchas de estas organizaciones se integran en Stop Trans Pathologization (STP), una plataforma internacional surgida en 2009 gracias a la iniciativa de grupos activistas procedentes mayoritariamente de España" ${ }^{12}$ En el año 2015, STP cuenta "con la adhesión de más de 397 grupos, redes activistas, instituciones públicas y organizaciones políticas" de los cinco continentes (STP 2015). La campaña STP centra actualmente sus esfuerzos en pedir la retirada de la categoría "Trastornos de la Identidad de Género" del capítulo V ("Trastornos mentales y del comportamiento") de la próxima edición de la CIE, cuya presentación está prevista para 2018. Partiendo del reconocimiento de que la despatologización y el derecho a una atención sanitaria son derechos humanos básicos y no excluyentes, STP propone la introducción de una mención no patologizante (como proceso de salud no basado en la enfermedad o trastorno) de la atención sanitaria a las personas "trans". Esta red internacional sugiere que el nuevo bloque de "atención sanitaria trans-específica" se incluya en el capítulo XXI: "Factores que influyen en el estado de salud y contacto con los servicios de salud". En la elaboración del nuevo bloque consideran fundamental realizar una descripción basada en los procedimientos relevantes para la atención sanitaria (y no en hipótesis etiológicas o criterios diagnósticos), reconocer la diversidad de trayectorias y garantizar el derecho a la cobertura pública de la atención (STP 2013; 2012; 2011).

\section{Reflexiones finAles: el ACCESO A LA SALUD COMO UN DERECHO FUNDAMENTAL}

Parece claro que no se puede despatologizar la transexualidad sin construir antes un marco normativo y político alternativo con el que garantizar la atención sanitaria. Ciertamente, la retirada sin más de los manuales podría poner en peligro el acceso a los recursos sanitarios, sobre todo en aquellos países en los que no existe un marco legal que garantice los derechos de las personas "trans"13. Con todo, el re- 
curso a los tratados internacionales que reconocen el derecho a la salud y bienestar de todas las personas, sin discriminación por motivos de orientación sexual o identidad de género, o la propuesta más pragmática de introducir una mención no patologizante en la próxima edición de la CIE, parecen alternativas lo suficientemente sólidas como para desiquiatrizar la transexualidad de los manuales de una vez por todas.

La libre expresión de las identidades de género ha de entenderse como un derecho fundamental, por lo que no puede estar sujeto a condicionamientos de índole clínica. En algunos países como Argentina, Dinamarca o Malta ya existen leyes que no exigen ningún requisito diagnóstico ni terapéutico para solicitar el cambio de sexo y de nombre en los documentos y registros oficiales, ya que se fundamentan en el principio de autodeterminación del propio género y en el derecho a la integridad corporal. En el contexto en el que se llevó a cabo esta investigación, Cataluña, la aprobación de la ley para la defensa de los derechos de las personas LGTBI y la erradicación de la LGTBI fobia ofrece el marco normativo necesario para la construcción de un modelo de atención sanitaria no basado en la enfermedad. El artículo 23.4 establece que "las personas transgénero y las personas intersexuales deben poder acogerse a lo establecido por la presente ley sin necesidad de un diagnóstico de disforia de género ni tratamiento médico" (Parlamento de Cataluña 2014).

La inclusión de la transexualidad en las clasificaciones de trastornos mentales, aparte de paradójica (pues la terapéutica no actúa sobre el supuesto trastorno mental, sino sobre el cuerpo), es contraproducente. A nivel individual, la persona diagnosticada puede interiorizar que su condición es patológica. A nivel social, el diagnóstico no ayuda en modo alguno a que entendamos que lo no es una disfunción o un error, sino una expresión más del ser humano. Considerar a estas personas como seres patológicos tiene un efecto social tranquilizante porque "sirve al resto de la sociedad para mantener su seguridad en su condición de género y desvía la atención sobre la dimensión política de la conflictividad de las normas de género" (Platero 2009: 126).

Además, hay que recordar que la inclusión de la transexualidad en los manuales clasificatorios de trastornos mentales no ha garantizado la atención sanitaria a estas personas. En muchos países sus demandas siguen siendo ignoradas; en otros, incluso, se persigue y condena a estas personas (Missé 2010). En España, no todas las comunidades autónomas financian la atención sanitaria trans-específica, y aquellas que sí lo hacen destinan tan pocos recursos que las listas de espera para someterse a las cirugías se cuentan en años.

En fin, ante las sucesivas reconceptualizaciones que han experimentado categorías como la "homosexualidad", el "travestismo" o la "transexualidad" en el DSM, fruto del ímpetu taxonomizador de la psiquiatría biomédica, resulta necesario problematizar la lógica reduccionista de las categorías diagnósticas referentes a las expresiones sexo-genéricas. Hay que despojarse "de esquemas mentales cerrados y pretendidamente seguros y atreverse a ahondar en las vicisitudes del deseo, haciéndose cargo de las incertidumbres que conlleva" (Garaizábal 2006: 169). Solo así estaremos en las mejores condiciones para entender que el deseo, la experiencia y los actos humanos desbordan el ideal homogeneizador y reduccionista de las nosologías sexuales.

\section{NOTAS}

[1] En España son nueve las comunidades autónomas que ofrecen atención sanitaria a las personas "trans", aunque no todas financian las cirugías genitales: Andalucia, Cataluña, Extremadura, País Vasco, Madrid, Comunidad Valenciana, Asturias, Aragón y Canarias (Armunia 2015).

[2] A lo largo del texto utilizaremos "trans" como una categoría paraguas con la que referirnos de forma conjunta a todas aquellas personas que expresan/experimentan un género distinto al asignado al nacer.

[3] Las UTIG son unidades hospitalarias creadas en aquellas Comunidades Autónomas españolas que han decidido financiar y supervisar el tratamiento hormono-quirúrgico para personas trans. Para poder acceder al tratamiento, la persona debe antes obtener un diagnóstico psiquiátrico que acredite su transexualidad.

[4] En España, la Ley reguladora de la rectificación registral de la mención relativa al sexo de las personas (2007) excluye la obligatoriedad de someterse a las cirugías de reasignación sexual para acceder al cambio de sexo administrativo, pero obliga a la persona a presentar un diagnóstico psiquiátrico y a seguir una terapia hormonal durante un periodo mínimo de dos años.

[5] Para acceder a estas sesiones fue necesario firmar un documento de confidencialidad con el que todos los presentes nos comprometíamos a no revelar lo dicho y acaecido durante la reunión. Por consiguiente, no han podido publicarse los datos obtenidos.

[6] Con el fin de preservar el anonimato de las personas entrevistadas, empleamos nombres ficticios, pero siempre respetando el género de los originales.

[7] A finales de los años 70 del siglo XX, Virginia Prince (1997) acuña por vez primera el término "transgénero" para referirse a la gente, como ella misma, que desea recurrir al tratamiento hormonal pero no a las cirugías genitales. Es Leslie Feinberg (2006) quien, en un panfleto de 1992, le da al término un significado parecido al actual.

[8] Durante el proceso de elaboración del DSM-5, la APA abrió un espacio en su web en el que cualquier persona podía realizar sus comentarios y sugerencias. Se recibieron en total más de 13.000 aportaciones que fueron trasladadas a los 13 grupos de trabajo (APA 2013b).

[9] Una vez publicada la versión final del DSM-5, se han eliminado los borradores de la página web de la APA.

[10] Estos apartados, que acompañan a bastantes categorías del manual, fueron incluidos por vez primera en el DSM-IV tras las críticas recibidas por la escasa atención que el DSM-III había prestado a los factores socio-culturales (Uribe 2000).

[11] El antiguo Secretario de Estado por la Igualdad y actual presidente del Partido Popular en Andalucía, José Manuel Moreno, "incluyó dentro de lo que se consideran 'derroches del sistema' los tratamientos de reasignación de sexo, comparándolos con la cirugía estética" (Moreno 2013). 
[12] El trabajo de dos organizaciones catalanas, la Guerrilla Travolaka y la Xarxa d'Acció Trans-Intersex, resultó determinante para la puesta en marcha de esta red internacional (Soley-Beltran y CollPlanas 2011).

[13] Al respecto, resultan de especial interés las críticas efectuadas por una trans colombiana, feminista y antropóloga, quien considera

\section{RefERENCIAS BibliogRÁficas}

American Psychiatric Association (APA). 2013a. "Gender Disphoria". Arlington, VA: American Psychiatric Association. Consulta 14 de febrero de 2016 (http://goo.gl/VR2fuX).

American Psychiatric Association (APA). 2013b. DSM-5. Diagnostic and Statistical Manual of Mental Disorders. Arlington, VA: American Psychiatric Association.

Armunia, C. 2015. "Así funciona una unidad de atención a persones transexuales en la sanidad pública". Eldiario.es, 10 de octubre. Consulta 14 de febrero de 2016 (http:// goo.gl/eqyDrM).

Asociación Psiquiátrica Americana (APA). 1989. DSM-III-R. Manual Diagnóstico y estadístico de los trastornos mentales. Barcelona: Masson.

Asociación Psiquiátrica Americana (APA). 2002. DSM-IV-TR. Breviario. Barcelona: Masson.

Asociación Psiquiátrica Americana (APA). 2014. DSM-5. Manual diagnóstico y estadístico de los trastornos mentales. Madrid: Editorial Médica Panamericana.

Austin, J. 1982. Cómo hacer cosas con palabras. Barcelona: Paidós.

Benjamin, H. 1966. The Transsexual Phenomenon. New York: Warner Books.

Braunstein, N. A. 2005. Psiquiatría, teoría del sujeto, psicoanálisis (Hacia Lacan). México: Siglo XXI.

Butler, J. 2006. Deshacer el género. Barcelona: Paidós.

Butler, J. 2007. El género en disputa. El feminismo y la subversión de la identidad. Barcelona: Paidós.

Cohen-Kettenis, P. y F. Pfäfflin. 2010. "The DSM Diagnostic Criteria for Gender Identity Disorder in Adolescents and Adults". Archives of Sexual Behavior 39: 499-513. http://dx.doi.org/10.1007/s10508-009-9562-y

Earle, J.W. 2014. "DSM-5". The Philosophical Forum 45: 179196. http://dx.doi.org/10.1111/phil.12034

Feinberg, L. 2006. "Transgender Liberation: A Movement Whose Time Has Come". Pp. 205-220 en The Transgender Studies Reader, editado por S. Stryker y S. Whittle. New York: Routledge. http://dx.doi. org/10.4324/9780203955055

Fisk, N. 1974. "Gender Dysphoria Syndrome. The Conceptualization that Liberalizes Indications for Total Gender Reorientation and Implies a Broadly Based MultiDimensional Rehabilitative Regimen". Western Journal of Medicine 120: 386-391.

Foucault, M. 2003. Historia de la sexualidad. La voluntad de saber. Buenos Aires: Siglo XXI.

Garaizábal, C. 2006. "Evaluación y consideraciones psicológicas". Pp. 163-174 en Ser transexual. Dirigido al paciente, a su familia, $y$ al entorno sanitario, judicial y social, editado por E. Gómez e I. Esteva. Barcelona: Glosa.

García Becerra, A. y M. Missé. 2010. "Diálogo trans-cultural". Pp. 197-204 en El género desordenado. Críticas en torno a la patologización de la transexualidad, editado por M. Missé y G. Coll-Planas. Madrid: Egales.

Hacking, I. 1999. "Making Up People". Pp. 161-171 en Science Studies Reader, editado por M. Biagioli. London: Routlegde. que el movimiento por la despatologización está impulsado por un "activismo empoderado que nos viene del Norte" que a menudo olvida la fuerte transfobia que impera en muchos países de la periferia globalizada (cf. García Becerra y Missé 2010).

Hammarberg, T. 2009. "Human Rights and Gender Identity". CommDH Issue Paper, Council of Europe Commissioner for Human Rights, Strasbourg. Consulta 14 de febrero de 2016 (http://goo.gl/iGQS09).

King, D. 1993. The Transvestite and the Transsexual: Public Categories and Private Identities. Aldershot, UK: Avebury.

Klerman, G. L. 1987. "Is the reliability of DSM-III a scientific or a political question?" Social Work Research and Abstracts 23: 3. http://dx.doi.org/10.1093/swra/23.4.3

Kupfer, D. J., E. A. Kuhl y D. A. Regier. 2013. "DSM-5.The Future Arrived". JAMA 309: 1691-1692. http://dx.doi. org/10.1001/jama.2013.2298

Langer, S. J. y J. I. Martin. 2004. "How Dresses Can Make You Mentally III: Examining Gender Identity Disorder in Children". Child and Adolescent Social Work Journal 21: 5-23. http://dx.doi.org/10.1023/ B:CASW.0000012346.80025.f7

Laungani, P. 2002. "Mindless psychiatry and dubious ethics". Counselling Psychology Quarterly 15: 23-33. http:// dx.doi.org/10.1080/09515070110102305

Lawrence, A.A. 2010. "Proposed Revisions to Gender Identity Disorder Diagnoses in the DSM-5". Archives of Sexual Behavior 39: 1253-1260. http://dx.doi.org/10.1007/ s10508-010-9660-x

Lawrence, A.A. 2014. "Gender Assignment Dysphoria in the DSM-5". Archives of Sexual Behavior 43: 1263-1266. http://dx.doi.org/10.1007/s10508-013-0249-z

Lev, A. I. 2013. "Gender Dysphoria: Two Steps Forward, One Step Back”. Clinical Social Work Journal 41: 288-296. http://dx.doi.org/10.1007/s10615-013-0447-0

Martínez-Guzmán, A. y M. Montenegro. 2010. "Narrativas en torno al Trastorno de Identidad Sexual. De la multiplicidad transgénero a la producción de trans-conocimientos". Prisma social. Revista de ciencias sociales 4: 1-44.

Martínez-Guzmán, A. y L. Íniguez-Rueda. 2010. "La fabricación del Trastorno de Identidad Sexual: Estrategias discursivas en la patologización de la transexualidad". Discurso y Sociedad 4(1): 30-51.

Martínez Hernáez, A. 2000. "Anatomía de una ilusión. EI DSMIV y la biologización de la cultura". Pp. 248-275 en Medicina y cultura. Estudios entre la Antropología y la Medicina, compilado por E. Perdiguero y J. M. ${ }^{\text {a Comelles. }}$ Barcelona: Edicions Bellaterra.

Mas Grau, J. 2015. "Transexualidad y transgenerismo. Una aproximación teórica y etnográfica a dos paradigmas enfrentados". Revista de Dialectología y Tradiciones Populares 70: 485-501. http://dx.doi.org/10.3989/ rdtp.2015.02.009

Mas Grau, J. 2013. "La patologización de la transexualidad. Un mecanismo legitimador de nuestro sistema dual de género". Quaderns de l'Institut Català d'Antropologia 18: 65-79.

Matte, N., A. Devor y T. Vladicka. 2009. "Nomenclature in the World ProfessionalAssociation for Transgender Health's Standards of Care: Background and Recommendations". International Journal of Transgenderism 11: 4252. http://dx.doi.org/10.1080/15532730902799979 
Mead, M. 2006. Sexo y temperamento en tres sociedades primitivas. Barcelona: Paidós Surcos.

Mejía, N. 2006. Transgenerismos. Una experiencia transexual desde la perspectiva antropológica. Barcelona: Edicions Bellaterra.

Missé, M. 2010. "La lucha por la despatologización trans y perspectivas de futuro del movimiento trans en BarceIona”. Pp. 205-214 en Jóvenes en la red. Anuario de movimientos sociales, editado por P. Ibarra y E. Grau. Barcelona: Icaria.

Missé, M. y G. Coll-Planas. 2015. "La identidad en disputa. Conflictos alrededor de la construcción de la transexualidad". Papers. Revista de Sociologia 100: 35-52. http://dx.doi.org/10.5565/rev/papers.637

Moreno, C. 2013. "El colectivo transexual, víctima de los ajustes sanitarios pese a las promesas del PP". Público, 23 de abril. Consulta 27 de febrero de 2016 (http://goo. $\mathrm{gl} / \mathrm{x} 632 \mathrm{k} 0)$

Nieto, J. A. 2008. Transexualidad, intersexualidad y dualidad de género. Barcelona: Edicions Bellaterra.

Nieto, J. A. 2011. Sociodiversidad y sexualidad. Madrid: Talasa.

Nieto, J. A. y J. de la Calle. 2015. "Identidades periféricas, acontecimiento identitario y derechos humanos de las personas "trans"género". Pp. 235-255 en Memorias, identidades y experiencias trans. (In)visibilidades entre Argentina y España, editado por J. L. Peralta y R. M. Mérida Jiménez. Buenos Aires: Biblos.

Parlament de Catalunya. 2012. "Declaració del Parlament de Catalunya contra l'homofòbia, la lesbofòbia i la transfòbia". Consulta 14 de febrero de 2016 (http://goo.gl/ OWi8bY)

Parlamento de Cataluña. 2014. "Ley 11/2014, de 10 de octubre, para garantizar los derechos de lesbianas, gais bisexuales, transgéneros e intersexuales y para erradicar la homofobia, la bifobia y la transfobia". Boletín Oficial del Estado 281: 94729-94748. Consulta 29 de febrero de 2016 (https://goo.gl/o1kt57).

Parlamento Europeo. 2011. "Resolución del Parlamento Europeo, de 28 de septiembre de 2011, sobre derechos humanos, orientación sexual e identidad de género en las Naciones Unidas". Consulta 14 de febrero de 2016 (http://goo.gl/pBKtZa).

Platero, R. L. 2009. "Transexualidad y agenda política: una historia de (dis)continuidades y patologización". Política y Sociedad 46: 107-128.

Platero, R. L. 2011. "The narratives of transgender rights mobilization in Spain". Sexualities 14: 597-614. http:// dx.doi.org/10.1177/1363460711415336

Plummer, K. 1995. Telling Sexual Stories: Power, Change and Social Worlds. New York/London: Routledge. http:/l dx.doi.org/10.4324/9780203425268

Prince, V. 1997. "Seventy Years in the Trenches of the Gender Wars". Pp. 469-476 en Gender Blending, editado por V. Bullough, B. Bullough y J. Ellias. New York: Prometheus Books.

Rorty, R. 1990. El giro linguístico: dificultades metafilosóficas de la filosofía lingüística. Barcelona: Paidós.

Soley-Beltran, P. 2013. "Transexualidad y transgénero: una perspectiva bioética". Revista de Bioética y Derecho 30: 21-39. http://dx.doi.org/10.4321/s188658872014000100003

Soley-Beltran, P. y G. Coll-Planas. 2011. "'Having words for everything'. Institutionalizing gender migration in Spain (1998-2008)". Sexualities 14: 334-353. http://dx.doi. org/10.1177/1363460711400811

Stoller, R. 1968. Sex and Gender. Vol. I, The Development of Masculinity and Femininity. Nueva York: Science House.

Stoller, R. 1975. Sex and Gender. Vol. II, The Transsexual Experiment. London: The Hogarth Press/The Institute of Psycho-Analysis.

Stop Trans Pathologization (STP). 2010. "Propuesta de petición a la American Psychiatric Association". Consulta 14 de febrero de 2016 (http://goo.gl/Xg0saM).

Stop Trans Pathologization (STP). 2011. "Reflexiones sobre despatologización trans y derechos sanitarios". Consulta 14 de febrero del 2016 (http://goo.gl/cMdTMa).

Stop Trans Pathologization (STP). 2012. "Reflexiones sobre el proceso de revisión de la $\mathrm{CIE}$ desde una perspectiva de despatologización y derechos humanos". Consulta 14 de febrero del 2016 (http://goo.gl/hcSvrE).

Stop Trans Pathologization (STP). 2013. "Desarrollos recientes relacionados con los procesos de revisión del DSM y de la CIE". Consulta 14 de febrero del 2016 (http:// goo.gl/m3nSxu).

Stop Trans Pathologization (STP). 2015. "Bienvenida". España: Stop Trans Pathologization. Consulta 27 de febrero de 2016 (http://stp2012.info/old/es).

Uribe, C. A. 2000. "La controversia por la cultura en el DSMIV". Revista colombiana de psiquiatría 29: 345-366.

Useche, B. 2005. "Medicalización, erotismo y diversidad sexual: una crítica sexológica al DSM-IV-TR". Sexología Integral 2: 87-95.

Valentine, D. 2007. Imagining Transgender: An Ethnography of a Category. Durham, NC: Duke University Press.

Valentine, D. y D. Kulick. 2001. "Transsexuality, Transvestism, and Transgender". Pp. 15888-15893 en International Encyclopedia of the Social \& Behavioral Sciences, editado por N. J. Smelser y P. B. Baltes. Amsterdam: Elsevier. http://dx.doi.org/10.1016/b0-08-043076-7/04009-2

Van Dijk, T. 1999. "El análisis crítico del discurso". Anthropos 186: $23-36$

Winters, K. 2008. Gender madness in American psychiatry: Essays from the struggle for dignity. Dillon, CO: GID Reform Advocates.

Wittgenstein, L. 1988. Investigaciones filosóficas. México / Barcelona: UNAM / Grijalbo.

JORDI MAS GRAU. Licenciado en Sociología y doctor en Antropología Social por la Universidad de Barcelona (España). Sus investigaciones se centran en el estudio de la diversidad sexual y de género, así como las violencias y normatividades sexo-genéricas. Actualmente, participa en una investigación coordinada por la Universidad de Barcelona sobre la LGTBfobia en ciudades pequeñas y medianas europeas. 